\title{
Diagnostic Imaging Findings and Endovascular Treatment of Patients Presented with Abdominal Pain Caused by Spontaneous Isolated Superior Mesenteric Artery Dissection
}

\author{
Abdala Maulid Mkangala ${ }^{1}$, Huimin Liang ${ }^{1,{ }^{*}}$, Xiang Jun Dong ${ }^{1}$, Yangbo $\mathrm{Su}^{1}$ and Luo Haohao ${ }^{1}$ \\ ${ }^{1}$ Department of Radiology, Union Hospital, Tongji Medical College, Huazhong University of Science and Technology, Wuhan, China \\ "Corresponding author: Department of Radiology, Union Hospital, Tongji Medical College, Huazhong University of Science and Technology, Wuhan, China. Email: \\ huiminliang39@gmail.com
}

Received 2019 May 11; Revised 2019 November 05; Accepted 2019 November 12.

\begin{abstract}
Background: Isolated dissection of visceral artery organs is very infrequently reported and when occurred mostly affected is the superior mesenteric artery (SMA) with abdominal pain as the commonest presenting features in symptomatic patients. Dissection can be detected by ultrasound and CT, but computed tomography angiography (CTA) is the best for demonstration of the true and false lumens of the lesion. Nonetheless, the perfect treatment has not been accepted yet. However, if left untreated, it is a lifethreatening condition.

Objectives: Our aim is to evaluate diagnostic imaging and endovascular treatment outcome of spontaneous isolated superior mesenteric artery dissection (SISMAD). Based on the angiographic configuration of SMA and location of dissection we will share our experience based on deployment of a bare straight stent, bare tapered stent, overlapping bare stent or coil assisting bare stent. Patients and Methods: Medical data from patients presented with symptomatic superior mesenteric artery dissection (SMAD) and had received endovascular treatment between January 2007 and December 2017 were extracted. Patient demographics, symptoms, diagnostic imaging, endovascular treatment, and follow-up findings were analyzed.

Results: Total of 31 patients were included in this study [87.1\% $(n=27)$ male, 12.9\% $(n=4)$ female, and mean age $52.9 \pm 8.2$ years]. All patients had abdominal pain as the main presenting symptom. The mean length of dissection was $(4.79 \pm 3.03) \mathrm{cm}$, mean distance from the aorta to dissection entry was $2.5 \pm 1.0 \mathrm{~cm}$, mean percentage stenosis was $63.3 \pm 12.7 \%$, Sakamoto type IIA was seen in $35.5 \%$ $(\mathrm{n}=11)$, and type IIB in 64.5\% $(\mathrm{n}=20)$. All of the patients received bare self-expandable stent whereby $90.3 \%(\mathrm{n}=28)$ received stent $(\mathrm{s})$ without coil, of which $64.3 \%(n=18)$ received single straight stent, $21.4 \%(n=6)$ received overlapping stent and $14.3 \%(n=4)$ received tapered stent. On the other hand, 9.7\% $(n=3)$ received coil assisting stent. Post-procedure normal blood supply to the distal SMA and relief of symptoms was noted. One hundred percent $(n=31)$ primary success rate was recorded during mean fasting and follow-up time of $4.9 \pm 1.9$ days and $15.5 \pm 4.8$ months, respectively.

Conclusion: Endovascular treatment with a bare stent is a safe, effective, and successful treatment for symptomatic SISMAD with satisfactory outcomes. We highly recommend it to be considered as a first-line treatment in severe co-morbidity patients who are unfit for open surgery.
\end{abstract}

Keywords: Abdominal Pain, Bare, CTA, Dissection, Endovascular, Stent, Superior Mesenteric Artery

\section{Background}

The superior mesenteric artery (SMA) is the 2nd of the three main anterior visceral blood vessels of the abdominal aorta that includes celiac and inferior mesenteric artery. Dissection of these vessels is very infrequently described and when occurred mostly affected is the SMA with abdominal pain as the commonest presenting symptoms among symptomatic patients (1-4). Nonetheless, in the present years, case reports of patients with spontaneous isolated superior mesenteric artery dissection (SISMAD) in which the involvement of the aorta is ruled out have been significantly increased (5-7). Due to advances usage of imaging know-how like multi-detector computed tomography (MDCT), proceeding into multiplanar reformation (MPR) along with reformation imaging and CTA have heightened detection of the acute stage of SISMAD (3). Currently enhanced CT is the preferred imaging in the examination of cases of acute abdominal pain in the emergency units. In cases of Isolated superior mesenteric artery dissection (ISMAD), CT has been described as useful for the 
first diagnosis and follow-up due to its ability to minimize the partial volume artifacts and reduce misdiagnosis of the artery (6). This is the results of the lengthwise orientation of SMA that located vertically to the studying plan $(8,9)$.

In general, computed tomography angiography (CTA) is the more accurate, non-invasive imaging method that is able to diagnose quickly especially in most cases of acute abdominal pain. The superiority of CTA is the fact that it is able to show a clear diagnosis of arterial dissection. It could show the length of dissection, extent, scope and involvement of the lumen. CTA could even further show true and false lumen, also indicate the presence of thrombosis and lumen stenosis if present. With the use of CTA, it is possible to analyze the involvement of important branches.

The illness is more prevalent in males in the 5 th decade of their life and generally situated about $1.5-3 \mathrm{~cm}$ from the aorta hence sparing the beginning of the artery (8). Etiology is still not yet well defined, though atherosclerosis, medial cystic necrosis, fibromuscular dysplasia, as well as untreated hypertension are reported to be risk factors (1, 9). The natural history of the illness is not clearly defined. In most cases, it depends on individual patients' presentations with specific clinical features. The common clinical presentation is abdominal pain, in particular acute or chronic epigastric pain, which is thought to be caused by the length of the lesion itself, intestinal ischemia or infarction with peritonitis $(4,10)$. Other presenting features are vomiting, nausea, diarrhea, and abdominal wall distention.

The treatment regimen is still not well-established, there are different approaches including conservative, endovascular, and open surgical treatment. Conservative treatment is used for patients with no sign of bowel ischemia (11, 12). For symptomatic patients, endovascular stenting and surgical repair have been reported. Currently, a significant number of articles describe endovascular stent treatment as the first treatment of choice in symptomatic patients with good clinical outcomes $(10,13)$. However, the debate still continues regarding the best choice in selecting a self-expandable bare or covered type of stent. Furthermore, there is the issue of whether to apply coil in the false lumen or not as well as the decision to use overlapping stents and tapered stents with complex vascular imaging configuration. To date, the best endovascular therapeutic strategy in symptomatic patients is not yet concluded.

\section{Objectives}

Herein, we report endovascular management outcome of 31 patients presented with abdominal pain who were treated with either a bare straight stent, bare tapered stent, overlapping bare stent or coil assisting bare stent. The goal was to investigate the clinical features, radiological findings, treatments, and prognosis of patients with symptomatic SISMAD. We will share our experience in endovascular treatment based on the angiographic geometrical configuration of SMA and location of dissection which may predict the successful outcome of the bare stent.

\section{Patients and Methods}

\subsection{Patients Demographic and Clinical Presentation}

This clinical research using retrospective medical record case review was allowed by the institutional review board, and hence the requirement for patient informed consent was waived. The hospital electronic database results were reviewed for all patients who had SISMAD diagnosis according to clinical and radiological imaging findings between 2007 and 2017. Patient demographics, clinical manifestation, associated risk factors, diagnostic imaging, treatment modalities, and outcomes were extracted by using a prepared review data table. All information and imaging of a patient with SISMAD presented with acute or chronic abdominal pain who underwent endovascular management with available follow-up CT data were extracted. The inclusion criteria were all patients with isolated lesions who underwent endovascular stenting. Exclusion criteria were patients who had isolated lesions without endovascular treatment procedure or those with lesions extended to the aorta.

Total of 66 patients with spontaneous SMA dissection who underwent endovascular treatment were systematically reviewed. Out of all patients, 35 had dissection that extended to the aorta and therefore were excluded from the study. Thirty one patients remained eligible for the study and were included for statistical analysis. All of our patients were symptomatic patients with all signs and symptoms related to SMA dissection with abdominal pain as the key presenting symptom. Patient flowchart selection is presented in Figure 1.

\subsection{Diagnosis}

In this study, diagnosis of SISMAD was reached through contrast-enhanced CT (Siemens definition AS 128 CT) and CTA. In all patients, contrast-enhanced CT scanning was performed with a thickness section of $1.5 \mathrm{~mm}$ and a pitch of 1 and CTA section scanning of $1.5 \mathrm{~mm}$ and a pitch of 1 . The constructive data thickness section was $0.75 \mathrm{~mm}$ with an increment of $0.5 \mathrm{~mm}$. Post-processing image methods used on the working station was three-dimensional (3D) volume rendering, multiplanar reconstruction, curved 


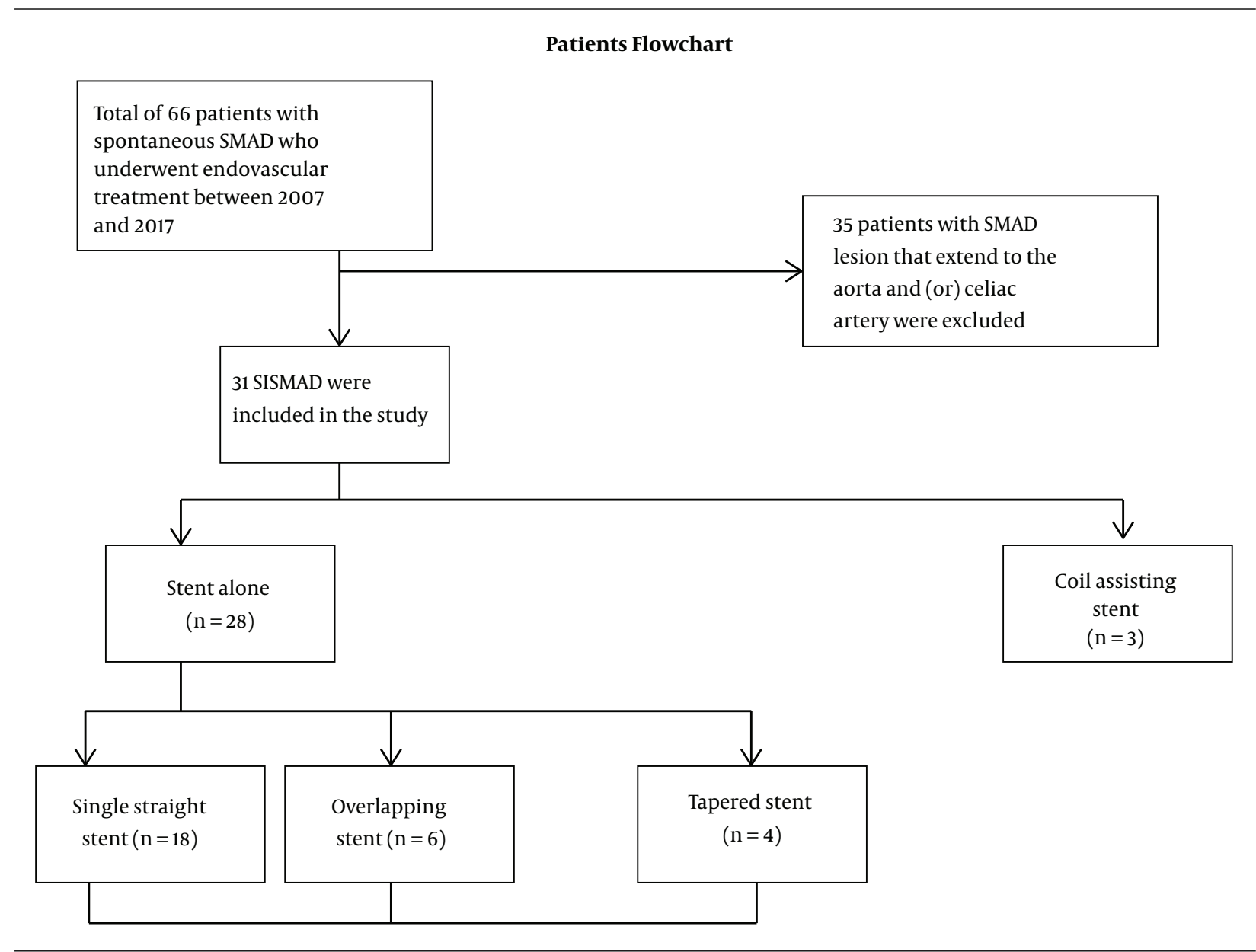

Figure 1. Patient flowchart selection (SISMAD, spontaneous isolated superior mesenteric artery dissection; SMAD, superior mesenteric artery dissection)

planar reconstruction and maximum intensity projection (MIP).

Contrast-enhanced CT was performed to establish the diagnosis of ISMAD, which was followed by CT angiography to establish the point of entry site, lesion length, and presence of false lumen or dissection/pseudo-aneurysm. The pathognomonic finding of SISMAD is the intimal flap (Figure $2 \mathrm{~A}$ ), and all cross-sectional modalities allow its identification. Furthermore, the longitudinal section from CTA imaging reveals the entry site, dissection length, and presence of pseudo-aneurysm (Figure 2B). On angiographic images (digital subtraction angiography-Siemens Artis Zee Celling and Siemens Artis Zee Floor, Siemens Medical Solutions, Muenchen, Germany, and Philips Allura Xper FD20 Philips Medical System, Best, The Netherlands) the SISMAD was proved by seeing the filling of the medium to the dissection sac with the same attenuation as its parent artery in the arterial phase (Figure $2 \mathrm{C}$ ). Filling defect detected between the parent artery and the false lumen sac indicated thrombus of the false lumen sac (Figures 2D and E). The proximal and distal arteries to the dissection as well as its branches were confirmed by CTA and digital subtraction angiography (DSA) images. Apart from SMA dissections, other radiological findings are hepatic cyst (Figure $2 \mathrm{~F}$ ), renal cyst, uterine fibroid, gall stone, accessory spleen, and pulmonary mass.

Sakamoto et al. (14) identified dissection lesions radiologically into four types based on contrast-enhanced CT scanning and false lumen appearance (type I-IV) which in turn does not account the fact that true lumen may somehow be compromised by thrombosis and result into stenosis. In 2009, Yun et al. (15) came up with modified classification, whereby based on radiological findings, in particular, the presence of true lumen patency and false luminal flow at the dissected segment, was categorized into three types. Type I, patent true and false luminal that show entry and re-entry sites; type II, patent true lumen but no reentry flow from the false lumen; IIA, visible false lumen but 

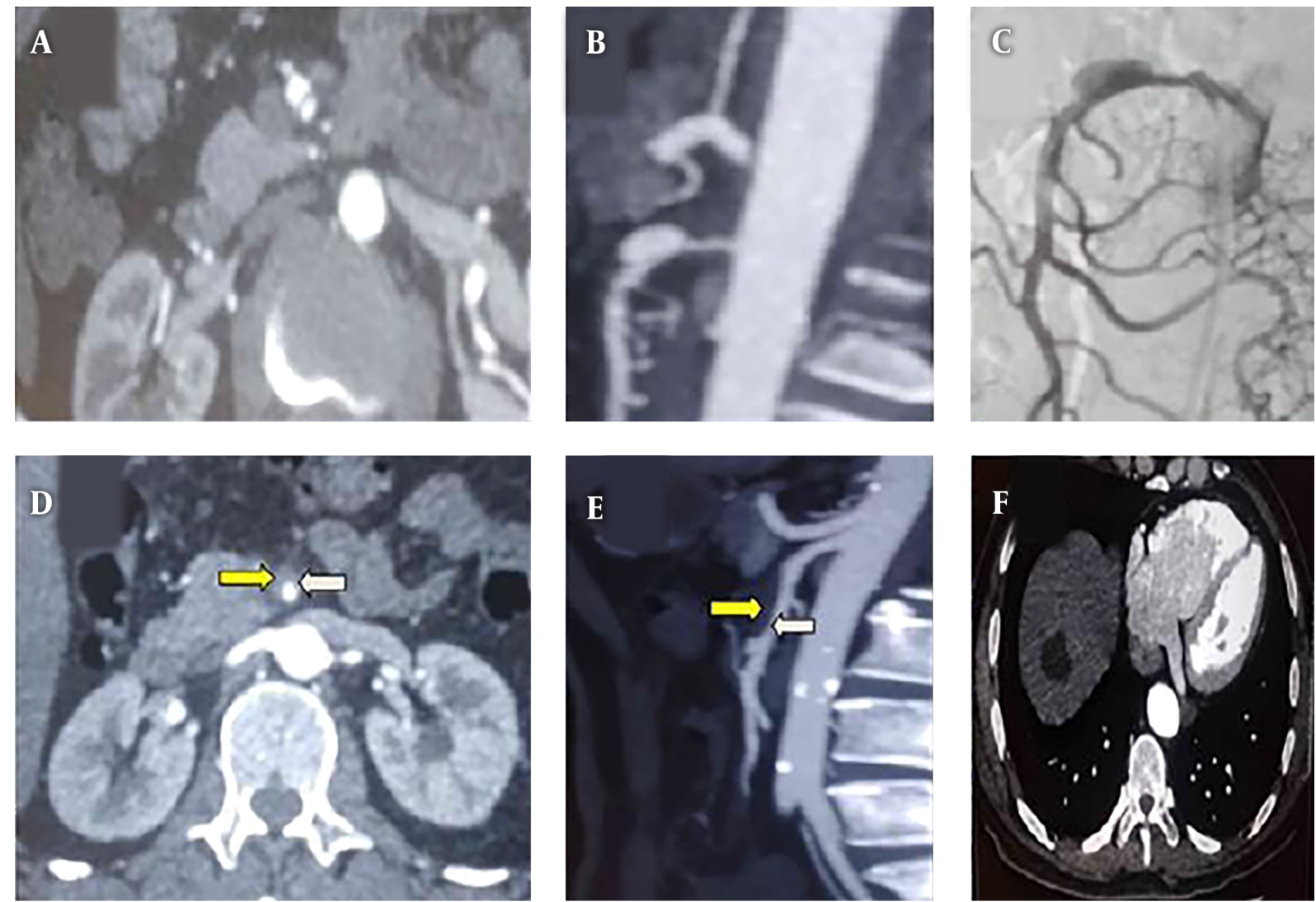

Figure 2. A, Abdominal CT angiogram, cross-section plane shows spontaneous isolated superior mesenteric artery dissection (SISMAD) with separated true and false lumen (intimal flapping- the pathognomonic finding of SISMAD); B, Computed tomography angiography (CTA), longitudinal section reveals the entry site, dissection length and presence of pseudo-aneurysm; C, Digital subtraction angiography (DSA) contrast medium filling the dissection sac with the same attenuation as its parent artery in the arterial phase; D, Abdominal CT angiogram, cross-section plane shows SISMAD with a separated true lumen (white arrow) and thrombosed false lumens (yellow arrow); E, CTA, longitudinal section shows attenuation differences between the parent artery (white arrow) and the false lumen (yellow arrow); F, CT image shows hepatic cyst.

no visible re-entry site (blind pouch of false lumen); IIB, no visible false luminal flow (thrombosed false lumen), which usually causes true luminal narrowing (Figure 2B); and III: SMA dissection with occlusion of SMA. In our study, we further noticed the need to analyze the geometry of the SMA and come-up with further subtypes whereby you have tapered and straight segment distal to the dissected segment which will determine whether to use straight or tapered stent as well as the application of coil in the false lumen.

\subsection{Treatment}

All of our patients underwent endovascular stent placement treatment after initial conservative management and observation failure. Endovascular procedures were performed by experienced interventional radiology with years of practice. They have much-needed knowledge and skills regarding clinical manifestation, dissection site and morphology, complications, and morbidities of the patients. The procedure was performed under the guidance of digital subtraction angiography through the Seldinger technique. Under local anesthesia, the right femoral artery was punctured by 18 gauge needle followed by introduction of 0.035 " $45 \mathrm{~cm}$ guidewire, then the needle was removed and $5 \mathrm{~F}$ catheter sheath with a dilator (Radifocus, Terumo Co., Tokyo, Japan). The dilator was removed and 5F Pigtail (Terumo) was introduced to the obtained aortogram in order to rule out other related vascular lesions. Once it was confirmed that it is SISMAD, the $8 \mathrm{~F}$ sheath was introduced. From the fact that SMAD lesion commonly occurs at about (1.5-3 cm) from the origin (8), to be safe, we used $8 \mathrm{~F}$ guiding catheter (Boston Scientific, Natick, Mass) to obtain selective SMA arteriogram in order to reveal the entry and the length of dissection as well as the proximal and distal flow of the vessel and dissection aneurysm morphology.

Once confirmed that the lesion is not so close to the origin of the SMA and the false lumen is not occluding the true 
lumen, then the interventional radiologist guided 0.035 " guidewire (Radifocus, Terumo Co., Tokyo, Japan) to cross the primary entry and get to the true distal lumen hence facilitating the pathway for stent deployment. The bare self-expanding stent was implanted over the opening site, typically starting from the distal to the proximal. Endovascular stenting treatments of SISMAD were performed using different stents (BIOTRONIK, MEDTRONIC, and BARD). Based on angiographic findings, we had four interventional treatment categories, straight stent, tapered stent, overlapping stenting and coil assisting stent. For lesions in which the dissection length was shorter than the primary stent and the proximal and distal SMA diameter was almost equal in measurements, single straight stent was used (Figure 3A ). For lesions with dissection lengths shorter than the primary stent and proximal diameters significantly larger than the distal diameter, single tapered stent was used (Figure 3B). In case the dissection length was larger than the primary stent, the second stent was introduced in overlapping stent fashion (Figure 3C). In some cases, the false lumen was significantly larger, therefore the microcatheter (Terumo) was guided to the neck of the false lumen and the coil (Cook Inc.) was introduced and packed in the false lumen cavity (Figure 3D).

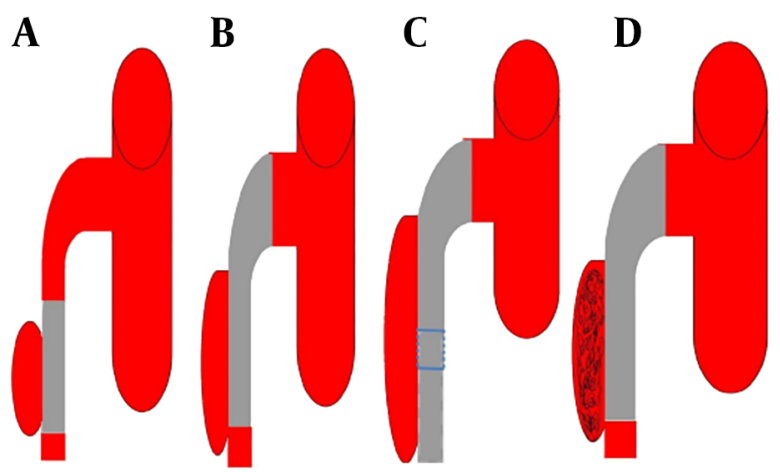

Figure 3. Four approaches we used in endovascular bare stent interventional treatment. A, Single straight bare stent; B, Single tapered bare stent; C, Overlapping bare stent; D, Coil assisting bare stent.

In all endovascular treatments, a pre-procedure 5000 IU bolus of heparin was administered. Angiography study contrast media (Omnipaque 350; Ge Health Care, Shanghai, China) were used. The volumes of $25 \mathrm{~mL}$ of contrast were injected at a flow rate of $5 \mathrm{~mL} / \mathrm{sec}$. The final angiogram was performed to confirm the position of the stent, full-length closure of the false lumen, and the distal blood flow. Finally, the femoral access site was closed with Perclose ProGlide (Abbott, Chicago, Ill). Post-procedure, all patients were kept fasting to continuously decompress the stomach (reduce upper abdominal pressure). They received anticoagulant, broad-spectrum antibiotics, gastric mucosal protection treatment, and intravenous fluids to maintain water and electrolyte balance. Nutritional support and close observation of patients for abdominal symptoms and vital signs were all provided.

\subsection{Follow-Up}

After the primary intervention, outpatient clinic attendances were insisted, where the complications, morbidity and mortality rates of treatment were recorded. Follow-up guidelines included history and clinical examination followed by CT angiography at 1, 6, and 12 months and yearly thereafter. Successful endovascular management was defined as a primary and secondary outcome. Primary success defined as normal blood supply to the distal SMA was restored, and symptoms were relieved. Secondary success is when the false lumen (pseudo-aneurysm) is obliterated with a patent stent on final follow-up CTA angiography. Patients were taken as a lost case during follow-up process if they missed two follow-up radiological studies after final procedures.

\subsection{Statistics}

In our study, descriptive statistics were used to report the various variables. Analyses were carried out by SPSS, version 15.0 (SPSS, Inc., Chicago IL). All of our continuous data were presented as mean \pm standard deviation (SD) and categorical data were presented as a percentage. In the current study due to limited data set in our disposal, the conduction and analysis of statistical tests were not carried out.

\section{Results}

The general demographic and clinical characteristics of 31 patients are summarized in Table 1 . Of these 31 patients, $87.1 \%(n=27)$ were male, and $12.9 \%(n=4)$ were female with the mean age of $52.9 \pm 8.2$ years (range, 38 - 68). All patients presented with abdominal pain $(100 \%, \mathrm{n}=31)$ that had either localized abdominal pain $(61.3 \%, n=19)$ or radiating pain to the back $(38.7 \%, \mathrm{n}=12)$. In some cases, the pain was associated with nausea $(54.8 \%, \mathrm{n}=17)$, vomiting $(54.8 \%, \mathrm{n}=17)$, and diarrhea $(29 \%, \mathrm{n}=9)$. The mean time for the onset of symptoms till admission to the hospital was $12.9 \pm 6.1$ days (range, 2 - 22). Relevant associated comorbidities included atherosclerosis in 61.3\% $(n=19)$, history of smoking in 51.6\% ( $n=16)$, hypertension in $38.7 \%$ ( $n$ $=12$ ), and diabetes mellitus in 6.5\% $(n=2)$.

About $87 \%$ of our cases were diagnosed as SISMAD by enhanced CT and 13\% were diagnosed by CTA. The mean 


\begin{tabular}{|c|c|}
\hline Variables & Values \\
\hline Age, $y$ & $52.9 \pm 8.2(38-68)$ \\
\hline \multicolumn{2}{|l|}{ Sex } \\
\hline Male & $27(87.1)$ \\
\hline Female & $4(12.9)$ \\
\hline \multicolumn{2}{|l|}{ Clinical symptoms } \\
\hline Duration, d & $12.9 \pm 6.1(2-22)$ \\
\hline Abdominal pain & $31(100)$ \\
\hline Localized abdominal pain & $19(61.3)$ \\
\hline Pain radiating to the back & $12(38.7)$ \\
\hline Nausea & $17(54.8)$ \\
\hline Vomiting & $17(54.8)$ \\
\hline Diarrhea & $9(29)$ \\
\hline Blood in stool & $0(0)$ \\
\hline \multicolumn{2}{|l|}{ Vascular risk factor } \\
\hline Atherosclerosis & $19(61.3)$ \\
\hline Smoking & $16(51.6)$ \\
\hline High BP & $12(38.7)$ \\
\hline Diabetes mellitus & $2(6.5)$ \\
\hline
\end{tabular}

Abbreviations: BP, blood pressure; d,day; SD, standard deviation; y, year ${ }^{\mathrm{a}}$ Values are expressed as No. (\%) or mean $\pm \mathrm{SD}$ (Range).

length of SMA dissection was $4.79 \pm 3.03 \mathrm{~cm}$ (range, 1.5 12.8). The mean length from the SMA origin to dissection entry point was $2.5 \pm 1.0 \mathrm{~cm}$ (range, 1.0 - 4.6). The mean percentage of stenosis was $63.3 \pm 12.7 \%$ (range, $43 \%$ - 95\%). Based on angiographic findings and SISMAD lesions, based on angiographic finding and considering Sakamoto classification, totally $35.5 \%(n=11)$ of patients showed type IIA lesion and $64.5 \%(n=20)$ showed type IIB lesion; no patient showed other types. Other radiological findings were hepatic cyst in 9.7\% $(n=3)$, renal cyst in 9.7\% $(n=3)$, uterine fibroid in $3.2 \%(n=1)$, gallstone in $3.2 \%(n=1)$, accessory spleen in $3.2 \%(n=1)$, and pulmonary mass in $3.2 \%(n=1)$.

All patients $(100 \%, \mathrm{n}=31)$ received endovascular stent placement treatment with a primary and secondary success rate of $100 \%$. All received bare self-expandable stent with a mean diameter of $7.1 \pm 0.5 \mathrm{~mm}$ (range, $6-8 \mathrm{~mm}$ ) and mean length of $57.3 \pm 14.4 \mathrm{~mm}$ (range, $20-100 \mathrm{~mm}$ ). All received bare self-expandable stent; whereby, $90.3 \%$ ( $n=$ 28 ) received stent $(s)$ without coil in which $64.3 \%(n=18)$ received single straight stent, $21.4 \%(n=6)$ received overlapping stent and $14.3 \%(\mathrm{n}=4)$ received tapered stent, while 9.7\% $(n=3)$ received coil assisting stent (Table 2$)$. Postprocedure DSA angiographic imaging shows true lumen restoration and normal blood supply to the distal SMA and its distal tributaries as well as relief of symptoms; therefore, $100 \%(n=31)$ primary success rate was achieved. No intra-operational or immediate post-operational complication was noticed. The mean fasting time was $4.9 \pm 1.9$ days (range, 2-6). All patients were successfully discharged home from the ward with no mortality recorded.

\begin{tabular}{|c|c|}
\hline Table 2. Angiographic Features and Endo & ent Outcome $(\mathrm{N}=31)^{\mathrm{a}}$ \\
\hline Variables & Values \\
\hline Dissection length, cm & $4.79 \pm 3.03(1.5-12.8)$ \\
\hline Ostium to dissection entry, cm & $2.5 \pm 1.0(1-4.6)$ \\
\hline Stenosis, \% & $63.3 \pm 12.7(43-95)$ \\
\hline \multicolumn{2}{|l|}{ Classification } \\
\hline IIA & $11(35.5)$ \\
\hline IIB & $20(64.5)$ \\
\hline \multicolumn{2}{|l|}{ Endovascular treatment } \\
\hline Length of stent, mm & $57.3 \pm 14.4(20-100)$ \\
\hline Diameter of stent, mm & $7.1 \pm 0.5(6-8)$ \\
\hline Bare self expandable & $31(100)$ \\
\hline Patient who received stent(s) only & $28(90.3)$ \\
\hline Single straight stent & $18(64.3)$ \\
\hline Overlapping stent & $6(21.4)$ \\
\hline Single tapered stent & $4(14.3)$ \\
\hline Patient received coil assisting stent & $3(9.7)$ \\
\hline Bare self-expandable stent & $31(100)$ \\
\hline Patent stent & $31(100)$ \\
\hline Duration of fasting, $d$ & $4.9 \pm 1.91(2-6)$ \\
\hline Duration of follow-up, mo & $15.5 \pm 4.8(10-26)$ \\
\hline Relief of symptoms & $31(100)$ \\
\hline
\end{tabular}

Abbreviation: d, day; mo, month; SD, standard deviation

${ }^{\mathrm{a}}$ Values are expressed as No. (\%) or mean $\pm \mathrm{SD}$ (Range).

All patients $(\mathrm{n}=31)$ were available during the follow-up period. The mean time of follow-up was $15.5 \pm 4.8$ months (range, 10 - 26). Patient post-interventional imaging results are available. Follow-up CTA shows visible SMA lumen patency, no displacement of the stent and the false lumen (pseudo-aneurysm) was obliterated, no endo-leak noted (Figure 4). No patients required further surgical intervention and all patients survived.

\section{Discussion}

In comparison to 1947, Bauersfeld first case report, SISMAD has recently been frequently reported due to the increased use of advanced technology in diagnostic imaging studies. Technological development, particularly in imaging diagnosis, lets us believe in the significant number of 


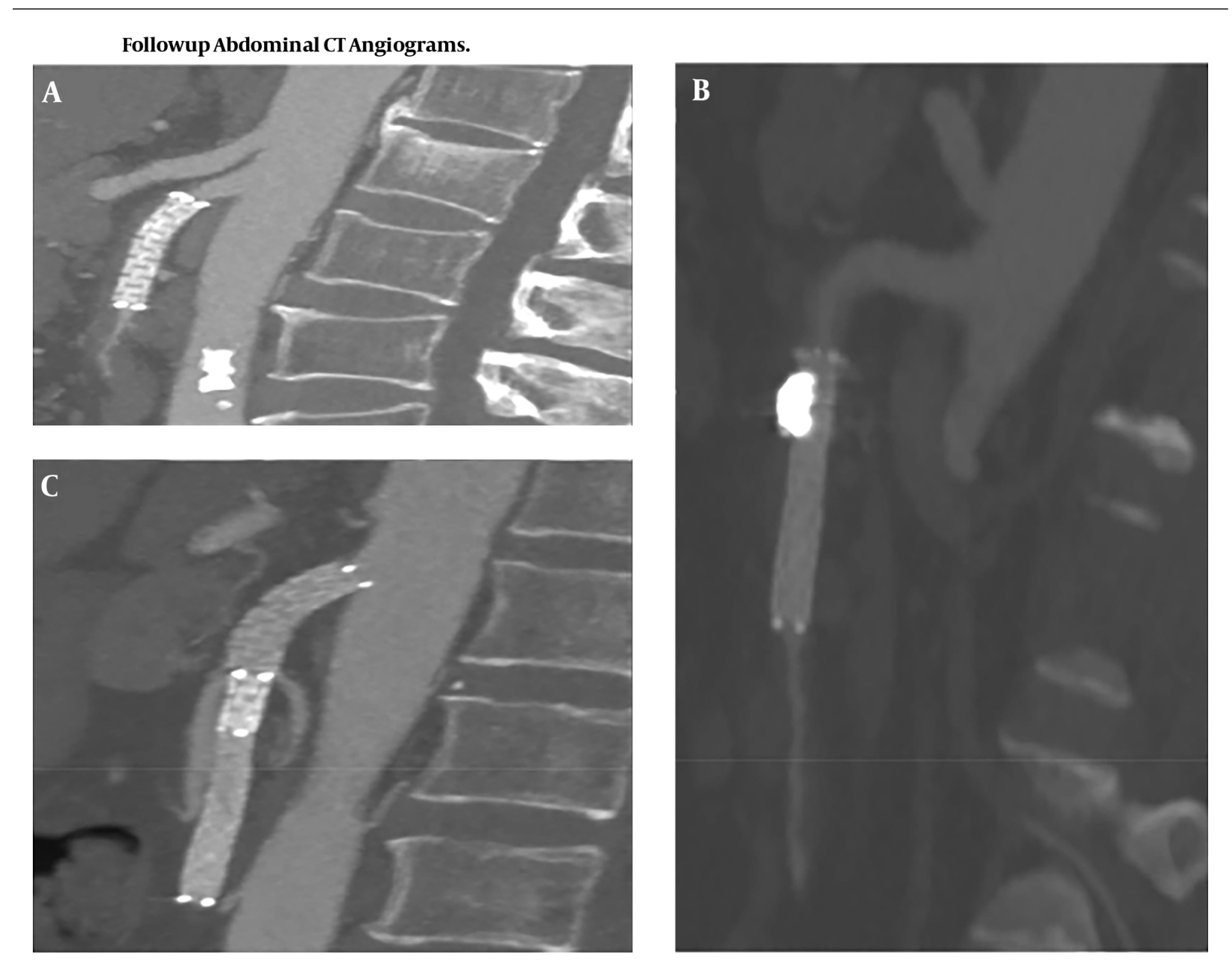

Figure 4. Follow-up abdominal CT angiograms, longitudinal section. A, Intact straight bare stent with no displacement; B, Intact coil assisting bare stent; C, Intact overlapping stent with no displacement.

cases reported every year. With this fact in mind, there is a need to establish a universal treatment regimen based on evidence-based findings in clinical practice with a significant number of patients. Many authors reported gender difference in the distribution of this disease mainly affecting males in the mean age of fifties of there life $(13,16)$. The patients who underwent endovascular treatment in our study were 27 male and four female with the mean age of 52.9 years that correlated with many findings.

SISMAD presentation could be described as symptomatic or asymptomatic according to abdominal pain. Abdominal pain is often associated with nausea, vomiting and sometimes diarrhea and passing bloody stool. In this study, all 31 patients presented with abdominal pain that presented as either localized abdominal pain or radiating to the back, but we did not find any patients with intestinal ischemia that makes us believe the dissection itself par- ticularly the length of dissection may had been the significant source of pain as it was reported in other findings where there was association of dissection and inflammation through stimulation of visceral nerve plexus.

Apart from intestinal ischemia, many other factors may play a role in abdominal pain such as aberrant hemodynamic strength due to the convex morphology of the SMA particularly at $1.5 \mathrm{~cm} 3 \mathrm{~cm}$ from the origin which may cause abdominal pain (17). This area of SMA is very important, especially in deciding the deployment of the stent. Similar to many vascular diseases, SISMAD is associated with risk factors such as smoking, diabetes, atherosclerosis, medial cystic necrosis, fibromuscular dysplasia, abnormalities of elastic fiber (Marfan syndrome and EhlersDanlos syndrome), trauma, as well as untreated hypertension $(1,4,9)$. Like another study, our patients presented with relevant associated vascular risk factors including hy- 
pertension, history of smoking, atherosclerosis and diabetes mellitus. No patients had an identifiable genetically related vascular disorder. In our findings, we hypothesize that both atherosclerosis and hypertension contribute significantly to the pathogenesis of dissection through destruction of vascular wall collagen and elastic fiber which in turn causes wall stiffness that results in dissection.

Currently, CTA is the preferred imaging modality in detecting and assessing SISMAD. Mural clot formation, intramural bleeding, intimal flap and enhanced attenuation around the SMA are a significant sign of SISMAD on CTA(18). The pathognomonic finding of SISMAD is the presence of intimal flap in cross-sectional imaging. In general, CTA is more accurate, non-invasive and will be able to diagnose quickly especially in most cases of acute abdominal pain. On the other hand, catheter angiography is more superior in assessing collateral circulation and the relationship of the lesion to branching vessels. Nevertheless, angiography may fail to show the lesion in case of SMA dissection in patients with a complete thrombosed false lumen (type III). Angiography is an invasive procedure; hence, this procedure should be preserved and used only to those patients with worsening symptoms, who need endovascular or surgical treatment. Our final patient diagnosis was based on CTA, and the confirmatory study was done during angiography imaging.

Based on angiographic findings found in the study conducted by Yun et al. (15), our patients belong to type IIA 11 and type IIB 17 with no type I or type III found. Solis et al. (8) hypothesis stated that usually dissection begins between $1.5 \mathrm{~cm}$ and $3 \mathrm{~cm}$ from the origin of the SMA, hence sparing the proximal origin of the artery. Our findings are similar to the hypothesis above whereby the mean distance of SMA ostium to the beginning of SMA dissection was $2.5 \mathrm{~cm}$ equally to the findings of the study performed by Solis et al. (8). Based on this, we highly recommend that in lesions that occur at this specific region, the stent should extend up to the origin and protect the convex curvature force that may cause stent migration. In case the lesion is further extended distally and the primary stent is not enough, the overlapping stent comes to play. The mean length of the SMA dissection was $4.79 \mathrm{~cm}$.

The treatment regimens are still not well-established; there is a different approach such as conservative, endovascular and open surgical treatment $(6,19-23)$. Conservative approach includes use of antiplatelet drugs, anticoagulants, control blood pressure and bowel rest. However recently risks and failures related to conservative treatment have been reported $(24,25)$ whereby there is report of patients developing recurrent clinical features and conditions worsening secondary to failure of the non-operative approach. These findings illustrate the treatment ap- proach need close follow-up. Though it does not prevent disease progression but should be considered as an option for some asymptomatic patients $(26,27)$. Endovascular and open surgery treatment generally are reserve options for the cases that abdominal pain does not subside, and there is clear evidence of signs indicative of bowel ischemia. In 2000, Leung et al. (28) reported the first successful case of SISMAD who was treated by endovascular stent placement using a wall stent. Subsequently, Froment et al. (29) came up with the recommendation in which endovascular stent treatment was proposed as a preferred treatment of choice. Their proposition was a result of the study they conducted in which they reported a failure rate of 38.5\% among 13 asymptomatic patients who received conservative treatment, which increases to $50 \%$ in symptomatic patients. Eventually, several authors started reporting stent placement as a safe, effective, and successful treatment in the management of symptomatic SISMAD. Recently, endovascular stent placement is reported as the first treatment choice with good clinical outcomes for the management of symptomatic patients or as a secondary treatment after conservative management failure $(9,12)$. Furthermore, the indications for endovascular treatment should not only be based on the presenting symptoms or percentage of true lumen occlusion but angiographic findings and the presence of collateral circulations through SMA side braches (30). In China, where our study originated, endovascular stent placement is considered as the first line of management for symptomatic patients. Hypothetically, in recent years, endovascular treatment has provided additional advantages compared to open surgery. Endovascular treatment is less invasive because of the reduced time of the healing process, the reduced time needed for immobilization and reduced infections. Overall, it is more sufficient in treating symptomatic patients with severe comorbidities who are unfit for open surgery $(6,16,21,22)$.

In our experience, the endovascular treatment was successful in all patients. We selected a flexible bare selfexpanding stent with a less radial force. This type of stent is suitable for the weak vascular wall and original curved site (31). A bare stent is sufficient enough in opening the true lumen and allowing normal flow through the distal part of the SMA and endothelialization of the stent with the formation thrombus in a false lumen. In this study, it was found that both bare stents alone (straight or tapered), overlapping stent or stent-assisted coiling showed significant success outcomes with long-term patency in aneurysm lesions. Furthermore, all clinical findings resolved rapidly following endovascular treatment. In our findings, we did not encounter any case of restenosis or stent migration and this shows the best way in selecting the stent is basically to use both straight stents in straight 
vessels and tapered stents in tapered vessels, as well as overlapping and coil, assisting stent in cases of long dissection and aneurysm dissection, respectively.

Our study emphasized that SISMAD should be included as one of the differential diagnosis among patients presenting with acute or chronic abdominal pain associated with nausea, vomiting or diarrhea who are in the 5th decade of their life. CTA is an ideal imaging modality in the investigation of these conditions. It would be effective in establishing the location of the entry site, dissection length, and presence of pseudo-aneurysm. The radiologist should consider the presence of intimal flap in crosssectional modalities as the key finding in establishing the presence of dissection. Endovascular stent placement is recommended in symptomatic patients especially in severe co-morbidity patients who are considered unfit for open surgery. The ideal choice of the stent should be a soft self-expandable stent, though it is more expensive. Radiological imaging morphology should dictate endovascular treatment especially whether to use a straight or tapered stent, overlapping stent or coil assisting.

Our study had several limitations. The study was a retrospective clinical case review with patients who received endovascular treatment. In addition, it was a singleinstitute study with a relatively small number of patients for conducting and analyzing statistical tests. Prospectively randomized clinical studies with a larger number of patients in collaboration will determine whether it is significantly different in the endovascular outcome between a patient with acute symptoms and those with chronic symptoms.

In conclusion, SISMAD is a rare vascular disease that presents with abdominal pain, nausea, vomiting, diarrhea or it may be asymptomatic. Recently it has become frequently reported due to the increased use of advanced technology in diagnostic imaging studies. Endovascular stent placement is a safe, effective, and successful treatment in the management of symptomatic SISMAD. Appropriate endovascular procedures to treat patients based on medical imaging results is a key point especially in patients with tapered vessels, a longer dissection lesion, and dissection aneurysm.

\section{Footnotes}

Authors' Contributions: Conception and design: Abdala Maulid Mkangala, Xiang-Jun Dong, Yangbo Su, Luo Haohao, and Huimin Liang. Analysis and interpretation: Abdala Maulid Mkangala, Xiang-Jun Dong, and Yangbo Su. Data collection: Abdala Maulid Mkangala, Xiang-Jun Dong, and Luo Haohao. Writing the article: Abdala Maulid Mkangala. Critical revision of the article: Abdala Maulid Mkan- gala, Xiang-Jun Dong, Yangbo Su, Luo Haohao, and Huimin Liang. Final approval of the article: Abdala Maulid Mkangala, Xiang-Jun Dong, Yangbo Su, Luo Haohao, and Huimin Liang. Overall responsibility: Abdala Maulid Mkangala.

Conflict of Interests: The authors declare that they have no conflict of interests.

Ethical Approval: The ethical approval was waived by the Institutional Review Board due to its retrospective nature.

Funding/Support: Financial or material supports were not received for this study.

\section{References}

1. Takayama T, Miyata T, Shirakawa M, Nagawa H. Isolated spontaneous dissection of the splanchnic arteries. J Vasc Surg. 2008;48(2):329-33. doi: 10.1016/j.jvs.2008.03.002. [PubMed: 18502087].

2. Mousa AY, Coyle BW, Affuso J, Haser PB, Vogel TR, Graham AM. Nonoperative management of isolated celiac and superior mesenteric artery dissection: Case report and review of the literature. Vascular. 2009;17(6):359-64. doi: 10.2310/6670.2009.00053. [PubMed: 19909685].

3. Zhao Y, Yin H, Yao C, Deng J, Wang M, Li Z, et al. Management of acute mesenteric ischemia: A critical review and treatment algorithm. Vasc Endovascular Surg. 2016;50(3):183-92. doi: 10.1177/1538574416639151. [PubMed: 27036673].

4. Clair DG, Beach JM. Mesenteric ischemia. $N$ Engl J Med. 2016;374(10):959-68. doi: 10.1056/NEJMra1503884. [PubMed: 26962730].

5. Luan JY, Li X. Computed tomography imaging features and classification of isolated dissection of the superior mesenteric artery. Eur J Vasc Endovasc Surg. 2013;46(2):232-5. doi: 10.1016/j.ejvs.2013.04.035. [PubMed: 23746739].

6. Gobble RM, Brill ER, Rockman CB, Hecht EM, Lamparello PJ, Jacobowitz GR, et al. Endovascular treatment of spontaneous dissections of the superior mesenteric artery. J Vasc Surg. 2009;50(6):132632. doi:10.1016/j.jvs.2009.07.019. [PubMed:19782510].

7. Okada M, Ishiguchi T, Itoh $\mathrm{H}$. Management of spontaneous dissection of the superior mesenteric artery. Intern Med. 2004;43(6):451-2. doi: 10.2169/internalmedicine.43.451. [PubMed: 15283177].

8. Solis MM, Ranval TJ, McFarland DR, Eidt JF. Surgical treatment of superior mesenteric artery dissecting aneurysm and simultaneous celiac artery compression. Ann Vasc Surg. 1993;7(5):457-62. doi: 10.1007/BF02002130. [PubMed: 8268091].

9. Sheldon PJ, Esther JB, Sheldon EL, Sparks SR, Brophy DP, Oglevie SB. Spontaneous dissection of the superior mesenteric artery. Cardiovasc Intervent Radiol. 2001;24(5):329-31. doi: 10.1007/s00270-001-25650. [PubMed: 11815839].

10. Li N, Lu QS, Zhou J, Bao JM, Zhao ZQ, Jing ZP. Endovascular stent placement for treatment of spontaneous isolated dissection of the superior mesenteric artery. Ann Vasc Surg. 2014;28(2):445-51. doi: 10.1016/j.avsg.2013.01.028. [PubMed: 24070572].

11. Park UJ, Kim HT, Cho WH, Kim YH, Miyata T. Clinical course and angiographic changes of spontaneous isolated superior mesenteric artery dissection after conservative treatment. Surg Today. 2014;44(11):20927. doi: 10.1007/s00595-014-0849-9. [PubMed: 24496981].

12. Park YJ, Park KB, Kim DI, Do YS, Kim DK, Kim YW. Natural history of spontaneous isolated superior mesenteric artery dissection derived from follow-up after conservative treatment. J Vasc Surg. 2011;54(6):1727-33. doi: 10.1016/j.jvs.2011.07.052. [PubMed: 21944909].

13. Kim JH, Roh BS, Lee YH, Choi SS, So BJ. Isolated spontaneous dissection of the superior mesenteric artery: Percutaneous stent 
placement in two patients. Korean J Radiol. 2004;5(2):134-8. doi: 10.3348/kjr.2004.5.2.134. [PubMed: 15235239]. [PubMed Central: PMC2698142].

14. Sakamoto I, Ogawa Y, Sueyoshi E, Fukui K, Murakami T, Uetani M. Imaging appearances and management of isolated spontaneous dissection of the superior mesenteric artery. Eur J Radiol. 2007;64(1):10310. doi: 10.1016/j.ejrad.2007.05.027. [PubMed: 17628380].

15. Yun WS, Kim YW, Park KB, Cho SK, Do YS, Lee KB, et al. Clinical and angiographic follow-up of spontaneous isolated superior mesenteric artery dissection. Eur J Vasc Endovasc Surg. 2009;37(5):572-7. doi: 10.1016/j.ejvs.2008.12.010. [PubMed: 19208448].

16. Javerliat I, Becquemin JP, d'Audiffret A. Spontaneous isolated dissection of the superior mesenteric artery. Eur J Vasc Endovasc Surg. 2003;25(2):180-4. doi: 10.1053/ejvs.2002.1785. [PubMed: 12552483].

17. Park YJ, Park CW, Park KB, Roh YN, Kim DI, Kim YW. Inference from clinical and fluid dynamic studies about underlying cause of spontaneous isolated superior mesenteric artery dissection. J Vasc Surg. 2011;53(1):80-6. doi: 10.1016/j.jvs.2010.07.055. [PubMed: 20855179].

18. Furukawa H, Moriyama N. Spontaneous dissection of the superior mesenteric artery diagnosed on multidetector helical CT. J Comput Assist Tomogr. 2002;26(1):143-4. doi: 10.1097/00004728-20020100000025. [PubMed: 11801921].

19. Iha K, Nakasone Y, Nakachi H, Horikawa Y, Gushiken M, Matsuda H. Surgical treatment of spontaneous dissection of the superior mesenteric artery: A case report. Ann Thorac Cardiovasc Surg. 2000;6(1):65-9. [PubMed: 10748364].

20. Nagai T, Torishima R, Uchida A, Nakashima H, Takahashi K, Okawara $\mathrm{H}$, et al. Spontaneous dissection of the superior mesenteric artery in four cases treated with anticoagulation therapy. Intern Med. 2004;43(6):473-8. doi: 10.2169/internalmedicine.43.473. [PubMed: 15283182]

21. Ishida M, Kato N, Hirano T, Suzuki T, Shomura Y, Yada I, et al. Dissecting aneurysm of the superior mesenteric artery successfully treated by endovascular stent-graft placement. Cardiovasc Intervent Radiol. 2003;26(4):403-6. doi: 10.1007/s00270-003-2639-4. [PubMed: 14667126].

22. Wu XM, Wang TD, Chen MF. Percutaneous endovascular treatment for isolated spontaneous superior mesenteric artery dissection: Report of two cases and literature review. Catheter Cardiovasc Interv.
2009;73(2):145-51. doi: 10.1002/ccd.21806. [PubMed: 19156877].

23. Tsai HY, Yang TL, Wann SR, Yen MY, Chang HT. Successful angiographic stent-graft treatment for spontaneously dissecting broadbase pseudoaneurysm of the superior mesenteric artery. J Chin Med Assoc. 2005;68(8):397-400. doi: 10.1016/S1726-4901(09)70183-2. [PubMed: 16138721].

24. Sparks SR, Vasquez JC, Bergan JJ, Owens EL. Failure of nonoperative management of isolated superior mesenteric artery dissection. Ann Vasc Surg. 2000;14(2):105-9. doi: 10.1007/s100169910019. [PubMed 10742422]

25. Morris JT, Guerriero J, Sage JG, Mansour MA. Three isolated superior mesenteric artery dissections: Update of previous case reports, diagnostics, and treatment options. J Vasc Surg. 2008;47(3):649-53. doi: 10.1016/j.jvs.2007.08.052. [PubMed: 18295120].

26. Nakamura K, Nozue M, Sakakibara Y, Kuramoto K, Satoh M, Kobayashi $\mathrm{S}$, et al. Natural history of a spontaneous dissecting aneurysm of the proximal superior mesenteric artery: Report of a case. Surg Today. 1997;27(3):272-4. doi:10.1007/bfo0941661. [PubMed: 9068114].

27. Yasuhara $\mathrm{H}$, Shigematsu $\mathrm{H}$, Muto T. Self-limited spontaneous dissection of the main trunk of the superior mesenteric artery. J Vasc Surg. 1998;27(4):776-9. doi: 10.1016/s0741-5214(98)70250-2. [PubMed: 9576098].

28. Leung DA, Schneider E, Kubik-Huch R, Marincek B, Pfammatter T. Acute mesenteric ischemia caused by spontaneous isolated dissection of the superior mesenteric artery: Treatment by percutaneous stent placement. Eur Radiol. 2000;10(12):1916-9. doi: 10.1007/s003300000520. [PubMed: 11305570].

29. Froment P, Alerci M, Vandoni RE, Bogen M, Gertsch P, Galeazzi G. Stenting of a spontaneous dissection of the superior mesenteric artery: A new therapeutic approach? Cardiovasc Intervent Radiol. 2004;27(5):529-32. doi: 10.1007/s00270-003-0158-y. [PubMed: 15461979].

30. Luan JY, Guan X, Li X, Wang CM, Li TR, Zhang L, et al. Isolated superior mesenteric artery dissection in China. JVasc Surg. 2016;63(2):5306. doi: 10.1016/j.jvs.2015.09.047. [PubMed: 26597665].

31. Zhang X, Sun Y, Chen Z, Li X. Therapeutic regimen options for isolated superior mesenteric artery dissection. Vasc Endovascular Surg. 2012;46(3):277-82. doi: 10.1177/1538574411434162. [PubMed: 22407428]. 